\title{
Avaliação de matrizes curriculares frente às DCN para os cursos de graduação em Odontologia
}

\author{
Lívia Maria Lopes de Oliveira*; Lívia Mirelle Barbosa*; Helder Lima Rebelo*; Thiago Coelho**; \\ Gustavo Pina Godoy***
}
* Mestre em Clínica Integrada, Programa de Pós-graduação em Odontologia, Universidade Federal de Pernambuco
** Mestrando em Clínica Integrada, Programa de Pós-graduação em Odontologia Universidade Federal de Pernambuco
*** Professor, Programa de Pós-graduação em Odontologia da Universidade Federal de Pernambuco

Recebido em 03/03/2019. Aprovado em 06/06/2019.

\begin{abstract}
RESUMO
O termo currículo refere-se ao percurso seguido pelo estudante em uma instituição de ensino superior desde o momento em que inicia seu processo de formação profissional até a graduação. As Diretrizes Curriculares Nacionais (DCN) para os cursos de graduação em Odontologia, instituídas pela Resolução CNE/CES 3, de 19 de fevereiro de 2002, definem os princípios, fundamentos, condições e procedimentos da formação de cirurgiões-dentistas, primando pela formação de um profissional integral. As Instituições de Ensino Superior (IES) têm em mãos o desafio de construir projetos pedagógicos que operacionalizem esse conceito, de modo a atender as demandas da sociedade. Neste contexto, este estudo de abordagem qualitativa e exploratória objetivou analisar criticamente as matrizes curriculares de dois cursos de Odontologia, pertencentes a IES localizadas em regiões distintas do país. A metodologia utilizada considerou um modelo lógico para avaliação da formação do cirurgião-dentista com base nas DCN, proposto por Pessoa e Noro. Foram analisadas as dimensões referentes à integração entre os ciclos básico e profissionalizante, ao desenvolvimento de competências e habilidades desejáveis, a formação profissional, além do desenvolvimento do estágio curricular e atividades complementares. Foi verificado que as duas estruturas curriculares estão em consonância com os ideais preconizados pelas DCN, apesar de conterem pontos que não fortalecem a formação profissional do egresso. Observou-se que ambas as matrizes apresentam potencialidades e fragilidades, que inspiram discussões.
\end{abstract}

Descritores: Recursos Humanos em Odontologia. Educação Baseada em Competências. Odontologia. Currículo. 


\section{INTRODUÇÃO}

O termo currículo refere-se ao percurso seguido pelo estudante em uma instituição de ensino superior (IES) desde o momento em que inicia seu processo de formação profissional até sua graduação. Sua construção, desde a promulgação da Lei de Diretrizes e Bases Nacionais da Educação (9394/96) - artigo $53^{1}$ é atribuição das universidades, dentro do exercício de sua autonomia. Para direcionar e, em certo aspecto, padronizar a formação superior no país, surgem as Diretrizes Curriculares, como legislação complementar, passando a ser publicadas a partir de $1998^{2}$.

As Diretrizes Curriculares Nacionais (DCN) para os cursos de graduação em Odontologia foram instituídas pela Resolução CNE/CES 3, de 19 de fevereiro de 2002. Estas definem os princípios, fundamentos, condições e procedimentos da formação de cirurgiões-dentistas, estabelecidos pela Câmara de Educação Superior (CES) do Conselho Nacional de Educação (CNE), para aplicação em âmbito nacional na organização, desenvolvimento e avaliação dos projetos pedagógicos dos cursos de graduação em Odontologia das IES ${ }^{3}$.

As DCN, na visão de Haddad et $a l_{.}{ }^{4}$, sinalizam para uma mudança paradigmática na formação de um profissional crítico, capaz de "aprender a aprender", de trabalhar em equipe, e de levar em conta a realidade social. A instituição formadora deve estar aberta às demandas sociais, $\mathrm{e}$ ser capaz de produzir conhecimento relevante e útil, priorizando a atenção à saúde universal e com qualidade, dando ênfase à promoção da saúde e prevenção das doenças. As Instituições de Ensino Superior (IES) têm, de acordo com mesmo autor, o papel de complementar o currículo de ensino com uma parte diversificada, capaz de refletir a experiência de cada instituição e as imposições do quadro regional em que se situa.

Nesse sentido, fica explicitado, pela legislação, o desafio imposto às IES para a construção coletiva de projetos que devem nortear a educação, abrindo espaço para que construam, a partir de um trabalho coletivo do colegiado docente, projetos em vários níveis, desde o Projeto Pedagógico Institucional à vida cotidiana da universidade, por meio dos quais os estudantes norteiem seu percurso 5 .

Historicamente, esse percurso era definido por meio de grades curriculares, nas quais as disciplinas eram organizadas em dois ciclos: o básico e o profissionalizante. $\mathrm{O}$ estudante tinha acesso aos estágios apenas no último ciclo. Esse modelo de ordenamento, após a instituição das DCN, passou a ser insuficiente, frente ao perfil desejável do formando colocado pela legislação. Esta visa ao desenvolvimento de um espírito crítico e reflexivo no graduando, além do domínio técnico-científico, de modo a instrumentalizá-lo para atuar com o novo, de maneira estrategicamente planejada e consciente, para a resolução de problemas do cotidiano das profissões, principalmente, no contexto do Sistema Único de Saúde ${ }^{4,6}$.

A formação profissional de nível superior não se limita apenas ao domínio de conhecimentos transmitidos em disciplinas de forma isolada, como era feito tradicionalmente. $\mathrm{O}$ conhecimento precisa ser adquirido de forma atrelada à realidade social, diante disso é proposta a organização curricular em forma de matriz articulada ou integrativa, possibilitando uma melhor inter-relação entre teoria e prática em torno de um eixo curricular voltado para a formação de profissionais mais humanos, éticos e reflexivos ${ }^{5,7}$.

A operacionalização desses conceitos representa uma tarefa árdua assumida pelas IES. De acordo com Fonseca ${ }^{8}$, a dificuldade das instituições de ensino de Odontologia em aderir às DCN reside no fato de que muitas funcionam mais como empresas do que como instituições formadoras e educativas. A preocupação em 
formar um profissional "socialmente engajado" extrapolaria, portanto, suas competências. O modelo tradicional, disciplinar, fragmentado seria para estas instituições, portanto, mais adequado.

Neste contexto, o objetivo deste estudo constitui em analisar criticamente duas matrizes curriculares (MC) de cursos de Odontologia de duas IES, tomando como referência as DCN.

\section{METODOLOGIA}

Trata-se de um estudo descritivo de abordagem qualitativa e exploratória, que utilizou análise documental ${ }^{9}$ para o alcance do objetivo proposto. Foi feita uma comparação de duas MC (MC1 e MC2) pertencentes a Cursos de Odontologia localizados em regiões distintas do Brasil com a resolução CNE/CES, de 19 de fevereiro de 2002. As MCs foram selecionadas de maneira aleatória.

A análise foi feita tomando como referencial uma adaptação do estudo de Pessoa e Noro $^{10}$, que propuseram um modelo lógico para avaliação da formação do cirurgião-dentista com base nas DCN (figura1).

As dimensões analisadas foram sumarizadas na tabela 1 , de modo a abranger todos os aspectos a serem considerados para a avaliação das matrizes 1 e 2 quanto ao atendimento das dimensões explicitadas nas $\mathrm{DCN}^{3}$.

\section{RESULTADOS E DISCUSSÃO}

As MCs contêm características distintas, referentes à organização do curso, distribuição da carga horária e conteúdo curricular (tabela 1).

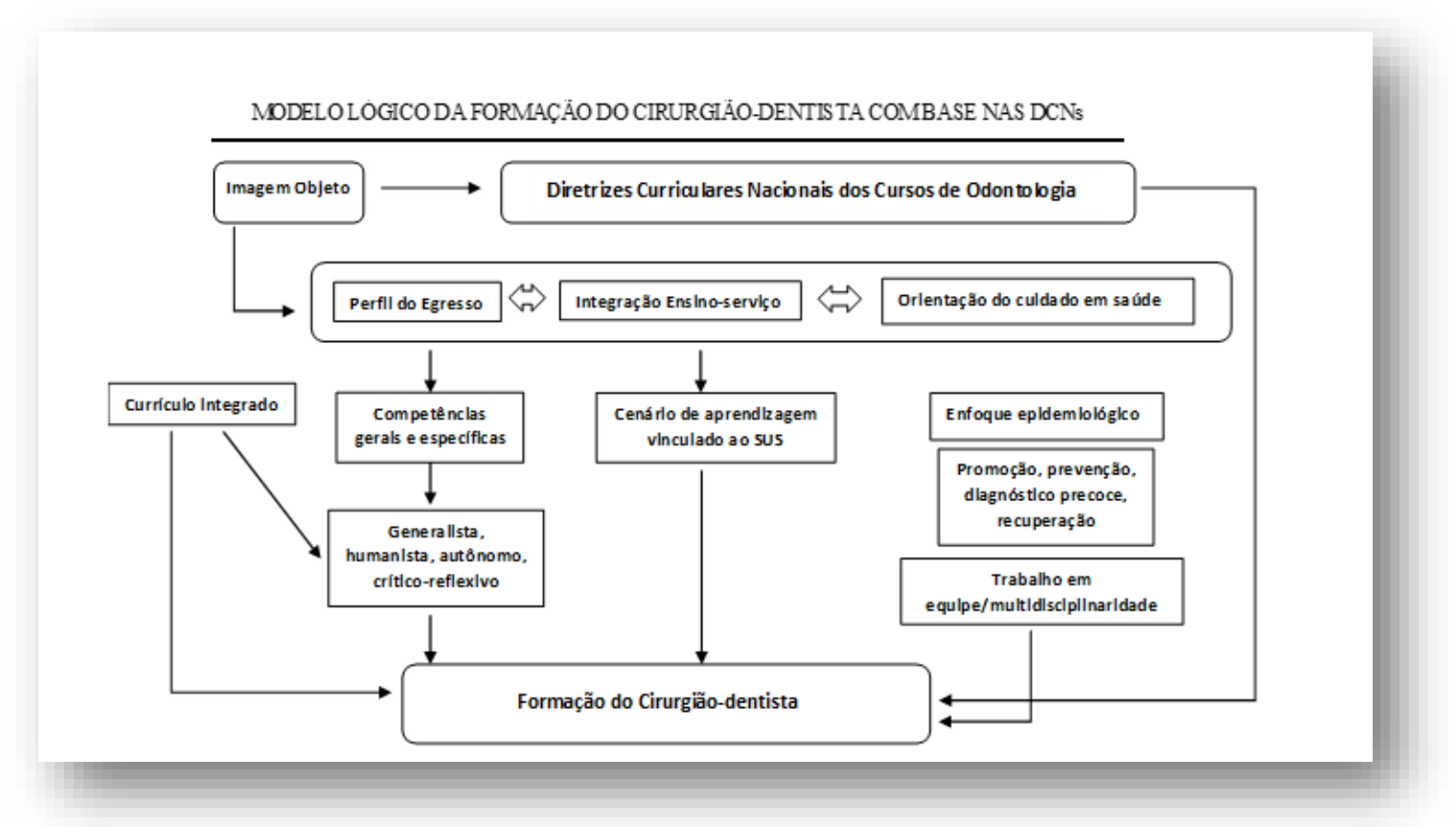

Figura 1. Modelo utilizado como base para a análise das matrizes curriculares selecionadas. Adaptado de Pessoa e Noro (2015) ${ }^{10}$ 
Tabela 1. Sumarização de aspectos característicos encontrados nas matrizes 1 e 2

\begin{tabular}{lcc}
\hline Característica avaliada & MC1 & MC2 \\
\hline Duração & 10 Períodos & 8 Semestres \\
Estrutura & Carga horária dividida entre & Sistema de créditos \\
teoria e prática & \\
Carga horária & 4140 horas & 4275 horas \\
Atividades Complementares & 100 horas $(2,4 \%)$ & 72 horas $(1,6 \%)$ \\
Atividades em rede & 100 horas $(2,4 \%)$ & Não \\
Estágio Supervisionado & 840 horas $(20,3 \%)$ & 648 horas $(15,1 \%)$ \\
Estágio em Saúde Coletiva & Não & 135 horas $(3,1 \%)$ \\
Total & 840 horas $(20,3 \%)$ & 819 horas $(19,1 \%)$ \\
Disciplinas Optativas (flexibilidade) & 60 horas $(1,44 \%)$ & 81 horas $(1,89 \%)$ \\
Conteúdo Curricular (disciplinas) & 3101 horas $(74,9 \%)$ & 3454 horas $(80,8 \%)$ \\
Tentativa de adequação à realidade local & Sim, disciplina de Educação e & Não \\
& Gestão Ambiental & \\
Metodologia da pesquisa & $4^{\circ}$ Período. & $1^{\circ}$ Semestre \\
Tentativa de integrar matérias básicas e & Não & Não \\
clínicas & & \\
\hline
\end{tabular}

Ambas as MCs estão em conformidade com a resolução $n^{\circ} 2$ da Câmara de Educação Superior do Ministério da Educação, que prevê uma carga horária mínima de 4000 horas para cursos de graduação em Odontologia ${ }^{11}$.

Nas MCs em análise observa-se ausência de integração entre os componentes curriculares dos ciclos básico e profissionalizante. A carga horária é prioritariamente conteudista, com atividades de estágios equivalendo a $20,3 \%$ na matriz 1 e 19,1\% na MC2. De acordo com as $\mathrm{DCNs}^{3}$, tais atividades devem ter carga horária mínima de $20 \%$ da carga horária total do curso.

Nas duas MCs o contato do estudante com atividades clínicas multi e interdisciplinares ocorre num período avançado do curso. Tais atividades poderiam ser iniciadas nos dois primeiros anos de curso, introduzindo o ensino em ambientes diversificados, como em serviços públicos de saúde voltados para a atenção básica. Isto possibilitaria ao discente o desenvolvimento de uma consciência dos problemas de saúde contextualizados socialmente, além de fortalecer a interação ensino-serviço para o desenvol- vimento de competências relacionadas à liderança e trabalho em equipe multidisciplinar, como preconizado pelas $\mathrm{DCN}^{7,12,13}$.

As disciplinas estão distribuídas de acordo com os eixos das DCN (Ciências Biológicas e da Saúde, Ciências Humanas e Sociais, Ciências Odontológicas) apesar de serem ofertadas de maneira fragmentada, à semelhança do currículo mínimo, historicamente posicionado há mais de 40 anos e que vigorou até o estabelecimento da LDB (Lei 9.394) em $1996^{14}$. A divisão do ensino entre os ciclos básico e profissionalizante é um resquício da Reforma do Ensino Superior de 1968, que as criou e instituiu os departamentos para uma maior racionalização de recursos. A formação mais voltada para a atividade clínica é uma manifestação da lógica desta fragmentação ${ }^{15}$. Faz-se necessário, entretanto, na visão de Poi et al. ${ }^{16}$ que o acadêmico compreenda que a doença não se inicia no indivíduo e nem termina no consultório, mas numa inter-relação complexa na qual interferem fatores econômicos, culturais, sociais e políticos.

As diretrizes preveem a formação de um 
profissional com competências e habilidades voltadas para atuação no serviço público, em resposta às demandas da sociedade, inferidas pelo levantamento epidemiológico de 2010 que comprovou uma elevada prevalência de doenças bucais e edentulismo na população brasileira ${ }^{17}$.

De acordo com as DCN, [...] a formação do cirurgião-dentista deverá contemplar o sistema de saúde vigente no país, a atenção integral da saúde no sistema regionalizado e hierarquizado de referência e contrarreferência e o trabalho em equipe de forma articulada ao contexto social, entendendo-a como uma forma de participação $e$ contribuição social - como exposto no Art. $5^{\circ}$, parágrafo único. ${ }^{3}$

Para tal, as diretrizes ainda indicam que deverá haver a formação de um profissional com habilidade para a tomada de decisões, que priorize o julgamento coletivo sobre o individual e que tenha desenvolvimento de competências gerenciais e de liderança dentro de equipes multiprofissionais. $\mathrm{O}$ componente curricular que poderia instrumentalizar o aluno para este fim seria a disciplina Saúde Coletiva, abordada de um ponto de vista transdisciplinar.

$\mathrm{Na} \mathrm{MC} 1$ a disciplina de Saúde Coletiva, com 120 horas de conteúdo teórico, é disponibilizada nos quatro períodos iniciais, junto às matérias básicas, quando o estudante não teve suficiente contato com matérias específicas das Ciências Odontológicas, para que possa desenvolver relacionamentos interdisciplinares de maneira mais eficiente. Percebe-se, ainda, pela ausência de tempo destinado às ações práticas, no ambiente do estágio supervisionado, que há uma falta de integração ensino-serviço, não possibilitando, portanto, o contato deste aluno com a realidade do setor público, o que dificulta o desenvolvimento das habilidades previstas nas diretrizes. Diferentemente do que ocorre na MC2 que apresenta uma maior carga horária teórica (162 horas) e prática em saúde coletiva incluindo estágio supervisionado na disciplina (135 horas), que favorece uma melhor qualificação do estudante para atuar no âmbito do Sistema Único de Saúde (SUS), permitindo que o perfil dos cirurgiões-dentistas egressos esteja em consonância com o preconizado pelas $\mathrm{DCN}^{3}$.

As DCN estão em alinhamento com os princípios do SUS e as Diretrizes da Política Nacional de Saúde Bucal. O SUS é um modelo de atenção integral e universal que abrange ações de promoção, prevenção, proteção e recuperação da saúde. A formação profissional deve, portanto, atender ao modelo vigente. Na visão de Fernandes et al. ${ }^{18}$, o egresso deve ser capaz de conhecer os fundamentos técnicos-científicos, sem fugir ao princípio da integralidade, adotando uma visão holística do paciente, inserido na complexidade que o cerca, privilegiando a odontologia como uma prática social.

A MC1, diferentemente da MC2, oferta a disciplina de Educação e Gestão Ambiental, talvez como um esforço de seus idealizadores no sentido de contribuir para a compreensão e difusão da cultura regional, presente nas DCN.

A MC1 disponibiliza a disciplina Metodologia Científica num período isolado do curso (quarto período), diferente da MC2 que a coloca em sua fase inicial, junto as matérias básicas. De acordo com as DCN, o profissional formado deverá estar dotado de conhecimentos a respeito de métodos e técnicas de investigação e elaboração de trabalhos acadêmicos e científicos. A oferta do conteúdo no início do curso, como na MC2, pode ser benéfica para o desenvolvimento de um espírito crítico-investigativo no aluno, desde que haja o reforço em períodos posteriores de sua trajetória. Este fato não ocorre em nenhuma das matrizes analisadas, visto que ambas dispõem de uma carga horária mínima que não proporciona, como consequência, um ambiente de estímulo para o desenvolvimento da pesquisa ao longo da graduação. Maltagliati e 
Goldenberg ${ }^{19}$ lançam luz, em sua pesquisa, sobre uma possível explicação para essas observações, destacando o papel da pesquisa na graduação, de acordo com as DCN: [...] A ampliação da rede do ensino superior, entre a Reforma Universitária e as vésperas da virada do século, não incorporou, em toda a sua extensão, a pós-graduação e o correspondente investimento em pesquisa institucional; tampouco as instituições privilegiam o espaço da instrução científica na graduação. À entrada do século XXI, as Diretrizes Curriculares, ao lado das propostas de ampliação de acesso, recomendam novas proposições de ensino-aprendizagem, junto à valorização da pesquisa e da responsabilidade social. Afirmando a flexibilidade da organização do currículo, as novas propostas preconizam reformulações curriculares que, em consonância com novas abordagens pedagógicas, ressaltem, para além $d a$ instrução científica, a consideração do ensino pela pesquisa ${ }^{19}$.

$$
\text { Com relação às disciplinas }
$$
complementares, necessárias à formação do perfil almejado para o egresso, a inserção institucional do curso, bem como flexibilidade individual, a MC1 oferta 100 horas para o desenvolvimento de atividades em rede (inespecificadas) e 100 horas para o desenvolvimento de atividades complementares. Não há como avaliar se esses espaços presentes na MC1 são preenchidos com ações que atendam ao que está estabelecido nas diretrizes. Há oferta de uma disciplina optativa (60h), sendo a escolha entre Informática Aplicada à Saúde ou Libras.

O limite de duas disciplinas oferecidas como optativas reduz de sobremaneira as opções do estudante, não favorecendo, portanto, a flexibilidade preconizada pelas DCN. Há consonância com o preconizado pelas DCN, neste ponto, uma vez que elas objetivam a formação de um profissional capaz de organizar, manusear e avaliar recursos de cuidado de saúde efetiva e eficientemente, além de desenvolver uma habilidade de comunicação, sem barreiras.

Nas palavras de Oliveira et al. ${ }^{20}$ [...] $a$ comunicação é um processo de interação no qual se compartilham mensagens, ideias, sentimentos e emoções. Na assistência em saúde, somente a partir de uma boa comunicação estabelecida se poderá identificar e resolver as necessidades dos pacientes de forma humanizada e integral. As instituições, especialmente as privadas, têm buscado atender à determinação legal e às recomendações do MEC quanto à adequação da matriz curricular dos projetos pedagógicos, inserindo a Libras como componente optativo, para atender ao perfil de profissionais humanistas, críticos e com uma atuação inclusiva.

A MC2 disponibiliza maior carga horária para disciplinas optativas $(81 \mathrm{~h})$, divididas em três opções com 27h. Não está, entretanto, especificado na matriz quais seriam as disciplinas optativas. Existe, no quarto semestre, a oferta de Língua Inglesa, apesar de não haver referência sobre a sua carga horária, nem ao tipo de oferta - se obrigatória ou optativa. Neste quesito, a MC2 encontra-se em consonância com as DCN, que preveem o domínio de pelo menos uma língua estrangeira por parte do graduando.

A ocorrência de uma carga horária semanal excessiva é percebida em ambas as matrizes, mais intensamente na MC2. Esta tem as 4.275 horas de sua carga horária total condensadas em 8 semestres, não sendo nela especificado quanto deste conteúdo refere-se a atividades práticas. Excesso de atividades curriculares implica em menor tempo para o aluno dedicar-se aos estudos.

De acordo com Lemos ${ }^{21}[\ldots]$ se não há tempo para estudo, também não há para a dúvida, a crítica, a reflexão e a mudança. Há espaço apenas para a reprodução de ideias e de técnicas. As aulas se tornam lócus de 
disseminação de resultados obtidos, informações $e$ verdades a serem repassadas, socializadas $e$ consumidas. A dimensão do trabalho intelectual perde-se e torna-se difícil alcançar o perfil do egresso proposto pelas diretrizes curriculares do curso de graduação: profissional crítico, reflexivo e transformador [...].

Quanto à avaliação das atividades clínicas das matrizes, pode-se observar que a MC1 oferta o componente curricular Clínica Integrada a partir do oitavo período se estendendo até o décimo período resultando em uma carga horária total de 400 horas de clínica, já a MC2 não apresenta Clínica integrada como componente curricular. A ausência desse componente na MC2 pode gerar uma deficiência na formação do egresso, pois a Clínica Integrada é responsável por resgatar a condição de totalidade e unicidade da prática odontológica, favorecendo a formação profissional generalista com sólida formação técnico-científica, humanística e ética, orientada para a promoção da saúde, com ênfase na prevenção de doenças bucais prevalentes, e também consciente da necessidade de educação continuada, conforme o preconizado nas DCN. ${ }^{22}$

Em nenhuma MC foi vislumbrada a existência de programas de fomento à pesquisa ou à extensão universitária. Essa tendência reflete um reducionismo do conceito de formação acadêmica. $\mathrm{O}$ foco exclusivamente no ensino, ou seja, transmissão de conhecimento, torna-se estéril, posto não estar associado a formação de um sujeito capaz de estabelecer uma compreensão ético-político-social, frente as diferentes realidades que o cercam. Há, dessa forma, desestímulo a um raciocínio problematizador, com habilidade para criar caminhos diante das dificuldades apresentas.

Nas MC é observada uma desassociação entre ensino, pesquisa e extensão, "a Santíssima Trindade". A indissociabilidade do ensinopesquisa-extensão deveria, de acordo com
Cordeiro e Andrade ${ }^{23}$, ser o princípio orientador da qualidade da produção universitária, uma vez que se traduz em um fazer universitário autônomo, competente e ético.

\section{CONSIDERAÇÕES FINAIS}

A implantação das DCN permitiu reformular as matrizes curriculares de cursos de Odontologia no Brasil. Entretanto, mudanças na legislação não bastam para mudar uma formação; analisar e repensar o papel da matriz curricular focalizando sua dinâmica, saberes e práticas devem ser metas entre os envolvidos no processo de formação dos cirurgiões-dentistas.

Diante da análise crítica das duas matrizes curriculares de cursos de Odontologia de diferentes instituições de ensino, tomando como referência as DCN, compreendeu-se que ambas as estruturas estão em consonância com os ideais preconizados pelas DCN, embora contenham pontos de fragilidade, principalmente, no tocante à integração ensino-serviço, assim como estímulo à pesquisa.

\section{ABSTRACT \\ Evaluation of curriculum norms according to the National Curriculum Guidelines for graduate studies in Dentistry}

The term "curriculum" refers to the program followed by students in an institution of higher learning from the moment they begin their course of professional training until graduation. The National Curricular Guidelines (NCG) for undergraduate studies in dentistry, instituted by Resolution CNE/CES 3, February 19, 2002, define the principles, basis, conditions and procedures for the training of fulltime professional dental surgeons. Higher Education Institutions (HEIs) face the challenge of designing pedagogical projects that implement these concepts in order to meet the demands of society. In this context, the objective of this qualitative and exploratory study was to critically analyze the curricular matrices of two dentistry 
programs from HEIs located in distinct regions of Brazil. A logical model based on national curriculum guidelines (NCG) proposed by Pessoa and Noro was used to evaluate the formation of dental surgeons. This study analyzed the integration between basic and professional activities, the development of desirable competencies and abilities, and professional development beyond residency and complementary activities. It verified that the curriculum structures of the two institutions are in agreement with the ideals advocated by the $\mathrm{NCG}$, in spite of containing aspects which do not strengthen the profession formation of graduates. It was observed that both models have strengths and weaknesses, which should elicit further discussions.

Descriptors: Dental Staff. Competency-Based Education. Dentistry. Curriculum.

\section{REFERÊNCIAS}

1. Brasil. Lei n $^{\circ}$ 9394, de 20 de dezembro de 1996. Estabelece as diretrizes e bases da educação nacional. Diário Oficial da República Federativa do Brasil. 1996, dez. 23; Seção 1. p 27833.

2. Catani AM, Oliveira JFDE. A educação superior. A organização do ensino no Brasil: níveis e modalidades na Constituição federal e na LDB. São Paulo, Brasil: Editora Xamã; 2002.

3. Brasil. Ministério da Educação. Conselho Nacional de Educação. Câmara de Educação Superior. Resolução CNE/CES, de 19 de fevereiro e 2002 [Institui Diretrizes Curriculares Nacionais do Curso de Graduação em Odontologia]. Diário Oficial da República Federativa do Brasil. 2002, 04 de mar; Seção 1:10.

4. Haddad AE, Morita MC, Pierantoni CR, Brenelli SL, Passarella T, Campos FE. Formação de profissionais de saúde no Brasil: uma análise no período de 1991 a 2008. Rev Saúde Pública. 2010;44(3):1-9.
5. Anastasiou LGC. Avaliação, ensino e aprendizagem: anotações para ações em currículo com matriz integrativa: Novas subjetividades, currículo, docência e questões pedagógicas na perspectiva da inclusão social. In: Encontro Nacional de Didática e Prática de Ensino, 2006; Recife. Pernambuco. P. 69-90.

6. Moreira COF, Dias MSA. Diretrizes Curriculares na saúde e as mudanças nos modelos de saúde e de educação. ABCS Health Sciences. 2015;40(3):300-5.

7. Emi DT, Silva DMC, Barroso RFF. Experiência do ensino integrado ao serviço para formação em Saúde: percepção de alunos e egressos de Odontologia. Interface (Botucatu). 2018;22(64):223-36.

8. Fonseca EP. As Diretrizes Curriculares Nacionais e a formação do cirurgião-dentista brasileiro. JMPHC. 2013; 3(2):158-78.

9. Sá-Silva JR, Almeida CD, Guindani JF. Pesquisa documental: pistas teóricas e metodológicas. RBHCS. 2009; 1(1):1-14.

10. Pessoa TRRF, Noro LRA. Caminhos para a avaliação da formação em Odontologia: construção de modelo lógico e validação de critérios. Ciênc Saúde Colet. 2015;20 (7):2277-90.

11. Brasil. Ministério da Educação. Conselho Nacional de Educação. Câmara de Educação Superior. Resolução $\mathrm{N}^{\circ}$ 2, de 18 de junho de 2007. Dispõe sobre carga horária mínima e procedimentos relativos à integralização e duração dos cursos de graduação, bacharelados, na modalidade presencial. [Internet]. Diário Oficial da República Federativa do Brasil. 2007; jun.02 [Acesso em 2019 fev 15]. Disponível em: http://portal. mec.gov.br/cne/arquivos/pdf/2007/rces002_0 7.pdf

12. Mendes R, Moura MS, Prado Jr RR, Moura LFAD, Lages GP, Gonçalves MPR. Contribuição do Estágio Supervisionado da 
UFPI para formação humanística, social e integrada. Rev Abeno. 2006;6(1):61-5.

13. Toassi RFC, Davoglio RS Lemos VMA. Integração ensino-serviço-comunidade: o estágio na atenção básica da graduação em Odontologia. Educ Rev. 2012; 28(4):223-42.

14. Ferrari MAMC, Araújo ME, Dias RB. A teoria na prática: proposta de curriculum frente às diretrizes curriculares nacionais do curso de graduação em Odontologia. Odonto. 2012; 20(39):17-26.

15. Lemos CLS, Fonseca SG. Saberes e práticas curriculares: um estudo de um curso superior na área da saúde. Interface (Botucatu). 2009;13(28):57-69.

16. Poi WR, Trevisan CL, Lucas LVM, Panzarini SR, Santos CLV. A opinião do cirurgiãodentista sobre a clínica integrada. Pesq Bras Odont Clín Int. 2003;3(2):47-52.

17. Roncalli AG, Côrtes MIS, Peres KG. Perfis epidemiológicos de saúde bucal no Brasil e os modelos de vigilância. Cad Saúde Pública. 2012; 28 (Supl): S58-S68.

18. Fernandes DC, Freitas DA, Pedrosa AK, Silva EN. Currículo de Odontologia e as Diretrizes Curriculares Nacionais. Rev Portal: Saúde e Sociedade. 2016;1(2):104-15.

19. Maltagliati LA, Goldenberg P. Reforma Curricular e Pesquisa na Graduação em Odontologia: uma História em Construção.
Hist Ciênc Saúde. 2007;14(4):1329-40.

20. Oliveira YCA, Costa GMC, Coura AS, Cartaxo RO, França ISX. A língua brasileira de sinais na formação dos profissionais de Enfermagem, Fisioterapia e Odontologia no estado da Paraíba, Brasil. Interface (Botucatu). 2012;16(43): 995-1008.

21. Lemos CLS. A implantação das Diretrizes Curriculares dos Cursos de Graduação em Odontologia no Brasil: algumas reflexões. Rev ABENO. 2001;5(1):80-5.

22. Almeida RVD, Padilha WWN. Clínica integrada: é possível promover saúde numa clínica de ensino odontológico? Pesq Bras Odontoped Clín Integr. 2000;1(3):23-30.

23. Cordeiro FMGS, Andrade F. Ensinopesquisa-extensão: um exercício de indissociabilidade na pós-graduação. Rev Bras Educ. 2009;14(41):269-80.

\section{Correspondência para:}

Gustavo Pina Godoy e-mail: gruiga@hotmail.com Av. Prof. Moraes Rego, 1235, Cidade Universitária 50670-901 Recife/PE 